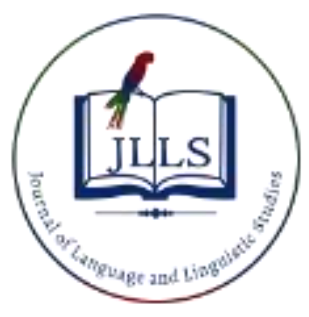

\title{
Errors in written expressions of learners of Turkish as a foreign language: A systematic review
}

\author{
Funda Uzdu Yıldız ${ }^{\text {a* }}$ (D), Betül Çetin b \\ ${ }^{a}$ Dokuz Eylül Üniversitesi, İzmir, Türkiye \\ ${ }^{b}$ Dokuz Eylül Üniversitesi, İzmir, Türkiye

\section{APA Citation:} \\ Uzdu Yıldız, F., \& Çetin, B. (2020). Errors in written expressions of learners of turkish as a foreign language: A systematic review. Journal of \\ Language and Linguistic Studies, 16(2), 612-625. \\ Submission Date:01/11/2019 \\ Acceptance Date:03/03/2020
}

\begin{abstract}
The present study systematically reviewed research that was conducted to analyze the errors within the written expressions of individuals learning Turkish as a foreign language. The sample of the study consisted of 16 articles that were identified through the review of literature. The articles that focused on the written expression errors made by learners of Turkish as a foreign language, published between 2010 and 2019, and written in Turkish with the participation of adult learners of Turkish as a foreign language were included in the study. The findings of the articles were assessed using content analysis to organize the systematic review. The results of the systematic review indicated that the errors that were most frequently made by foreign students in writing were related to spelling and punctuation $(40 \%)$, followed by grammatical errors (34\%). While lexical errors constituted $14 \%$ of errors, syntactic errors constituted $12 \%$ of the errors. At all levels, lexical and syntax errors were fewer than other types of error.
\end{abstract}

(C) 2020 JLLS and the Authors - Published by JLLS.

Keywords: Turkish as a foreign language; error; error analysis; systematic review; written expression

\section{Introduction}

The depiction of the errors made by foreign language learners can be instrumental in facilitating teaching and making it effective and efficient as such depictions show the areas in which most errors were made and reveal the causes of errors. Determination of the causes of errors is crucial for a more systematic organization of the efforts to minimize or eliminate the errors. To this end, analysis of the errors made by language learners commenced and underwent various changes over time, in line with theories of learning. Until the 1960s, through a behaviorist approach, it was assumed that native language might play a role in turning the errors made by language learners into habits. Therefore, comparisons were made between native language and target language to identify the causes of errors. In later periods, the direction of research changed as the view that errors might not occur solely under the influence of the native language was accepted and new theories of learning were developed (thanks to

\footnotetext{
${ }^{*}$ Corresponding author. Tel.: +90-232-463-0267

E-mail address: funda.uzdu@deu.edu.tr
} 
the improvement of cognitive processes through experimental studies) in the wake of behaviorist learning theories. Error analysis approaches were adopted in addition to the contrastive analysis that is related to native language. The two complementary approaches were evaluated separately or together. The studies by Pit Corder, who is accepted as a foundational researcher in the area of error analysis, are trendsetters in this field. Corder (1981) uses the term "error analysis" and İmer, Kocaman, and Özsoy (2011, p. 318) offer "yanlış çözümlemesi" as the Turkish equivalent of it. Corder (1981, p. 174) highlights that the main purpose of error analysis is to identify what students know and what they do not and to ensure that rules in the target language are arranged in a more effective manner with the right information and data by showing the instructor as to which assumptions are wrong. Corder (1981, p. 35) argues that error analysis is evidently beneficial to teachers as it provides them with feedback, and this feedback provides teachers with the opportunity to review and improve their teaching materials, methods, and course content. For Sridhar (Fisiak 1981, p. 225), another researcher who had studies in this field, analysis of the errors made frequently by learners contributes to the process of arranging the topics to be covered in the classroom and in textbooks from easy to difficult; selecting the topics with which to test learners' competency; and organizing teaching practices as well as remedial courses. There are certain methods that are developed by researchers on how this analysis - aims and benefits of which have been described above — should be conducted. These methods are presented in Table 1 .

Table 1. Error Analysis Methods

\begin{tabular}{ll}
\hline \multirow{3}{*}{ Corder $(1967,1973)$} & 1. Collection of sample errors \\
& 2. Identification of errors \\
& 3. Description of errors \\
\hline 1. Collection of data & 2. Identification of errors \\
3ridhar (1981) & 3. Classification into error types \\
& 4. Statement of relative frequency of error types \\
& 5. Identification of areas of difficulty \\
& 6. Therapy remedial drills, lessons, etc. \\
\hline 1. Collect data & 2. Identify errors \\
3. Classify errors & 4. Quantify errors \\
& 5. Analyze source \\
6. Remediate \\
\hline Khansir ve Ahrami (2014)
\end{tabular}

As seen in Table 1, the first step is data collection. The data are structures produced by students, and these structures may change depending on whether they are taken from verbal or written products. This change is important in determining the next step. One of the most frequently used methods for identifying and analyzing errors is to focus on the errors in written expressions. Certain usages, which might not be considered as errors in verbal products, may be accepted as errors in written products. In this sense, the first step is decisive for other steps. The second step is where errors are defined and described and every usage that is to be accepted to be erroneous is defined in this step. The next step requires the classification of the errors and the determination of their frequencies. 
The aim of this study is to collectively assess the findings of the studies that examined the errors made by learners of Turkish as a foreign language in their written expressions and to determine whether errors differed on the basis of various variables. The purpose of this analysis was to identify the general distribution of the errors made by learners of Turkish as a foreign language. Studying the errors made by foreigners while learning Turkish is important in that it demonstrates the overall frequency of errors that occur despite learner differences. It is believed that such findings will be beneficial for curricula. There are many articles written on the errors that learners of Turkish as a foreign language make in their written expressions (e.g., Bölükbaş, 2011; Ak Başoğul \& Can, 2014; Boylu, 2014; Çetinkaya, 2015). Those articles follow similar methodologies to study errors, and an examination of their findings taken all together will be useful to see the big picture. In his study entitled "Yabancılara Türkçe Öğretimi Alanında Yayınlanan Makaleler Üzerine Bir Analiz" [An Analysis Study On The Published Articles Relating To The Field Of Teaching Turkish To Foreigners], Biçer (2017, pp. 240-241) focused on the studies conducted between 2010 and 2016 and found the rate of error analysis studies among those studies to be $3.1 \%$. She reported the rates of studies on teaching grammar and teaching vocabulary was $12 \%$ and $15 \%$, respectively. The studies generally focused on the examination of errors while they varied on the basis of a specific country/language. In the present study, the country or language differences were not considered to be a distinguishing criterion, and all studies that generally dealt with the errors made in Turkish were included in the scope. A review of literature was carried out to identify the studies published on the errors that learners of Turkish as a foreign language make in their written expressions, and the articles were collated to evaluate the data on this topic.

\section{Method}

Following the review of literature, relevant articles were collated to collect data on the written expression errors made by learners of Turkish as a foreign language. The methodological design of this study was selected as meta-analysis, which is a qualitative research method. Introduced by Glass (1976), the term meta-analysis is defined as the "analysis of analyses." This term is described as the process by which "findings from individual studies conducted with a specific purpose are collected and a statistical analysis is performed on them" (1976, p. 3). Meta-analysis studies are studies wherein results of previous studies with the same or related aims are brought together to generate more generalizable results that are confirmed by multiple studies (Büyüköztürk et al., 2015, p. 229). To this end, a review of literature is initially conducted to identify the studies related to the topic under investigation. Then, the findings from these studies are collected for re-analysis. Thus, the findings of the studies previously conducted on a specific topic are obtained in a holistic approach. However, in the present study, "generalizing and encoding the results," a step required for meta-analysis, could not be performed because of the differences in language levels of sample groups and error analysis classifications of the related studies. Although the steps of the meta-analysis methodology were adopted, generalizations could not be performed to determine the differences in statistical results. Therefore, the study was transformed into qualitative research, and the data were evaluated through a systematic review. Qualitative research aims to describe the subject-matter realistically in its natural environment by using various data collection methods, including observation, interviewing, and document analysis (Yıldırım \& Şimşek, 2013). Karaçam $(2013$, p. 26) indicates that systematic reviews have grown in popularity in recent years as these studies "combine findings from multiple studies on a specific topic and generate the best evidence through critical analysis." Content analysis is used for analyzing the data. As noted by Çalık and Sözbilir (2014, p. 36), the general purpose of analysis in the field of education and instruction is to save studies from "repetition and clutter" and ensure that "teachers and researchers who wish to monitor the 
developments in educational research despite their workload and difficulties in accessing those studies" can obtain information.

\subsection{Data collection procedures}

The articles that focused on the written expression errors made by learners of Turkish as a foreign language, published between 2010 and 2019, and written in Turkish with the participation of adult learners of Turkish as a foreign language were included in the study. The search made using the keywords "hata çözümlemesi," "yanlış çözümlemesi," and "error analysis" resulted in a total of 34 articles written on teaching Turkish as a foreign language, and one article was excluded because its participants consisted of refugees in Turkey who were not "learners of Turkish as a foreign language." Three articles were excluded as they focused on phonology and verbal language. Fourteen articles were excluded because of their dealing exclusively with errors on morphology or grammar, which was considered to potentially lead to changes in rates of findings in general. The steps used for the selection of the articles are presented in Table 2 .

Table 2. Selection of articles

\begin{tabular}{cll}
\hline Identification & Articles accessed & $(\mathrm{n}=34)$ \\
\hline Separation & Nature of the study group & $(\mathrm{n}=1)$ \\
\cline { 2 - 3 } & Study of verbal language and phonology & $(\mathrm{n}=3)$ \\
\cline { 2 - 3 } & Study of special issues & $(\mathrm{n}=14)$ \\
\cline { 2 - 3 } & Suitable for the current topic & $(\mathrm{n}=16)$ \\
\hline Suitability & Suitable for inclusion in the review & $(\mathrm{n}=16)$ \\
\hline
\end{tabular}

The articles which were found suitable for inclusion in the review are presented in alphabetic order in Table 3.

Table 3. Articles Found Suitable for Inclusion in the Review

\begin{tabular}{|c|c|c|c|c|c|}
\hline Autor(s) & Year & Title of The Article & Journal & Vol & Pages \\
\hline $\begin{array}{l}\text { Ak Başoğul, } \\
\text { D. ve Can, } \\
\text { F.S. }\end{array}$ & 2014 & $\begin{array}{l}\text { Yabancı Dil Olarak Türkçe Öğrenen } \\
\text { Balkanlı Öğrencilerin Yazılı Anlatımda } \\
\text { Yaptıkları Hatalar Üzerine Tespitler } \\
\text { [The Determination about the Mistakes } \\
\text { in the Explanation of the Balkan } \\
\text { Students Learning Turkish as a Foreign } \\
\text { Language] }\end{array}$ & $\begin{array}{l}\text { Dil ve Edebiyat } \\
\text { Eğitimi Dergisi } \\
\text { [Journal of Language } \\
\text { and Literature } \\
\text { Education] }\end{array}$ & 10 & $100-119$ \\
\hline Boylu, E & 2014 & $\begin{array}{l}\text { Yabancı Dil Olarak Türkçe Öğrenen } \\
\text { Temel Seviyedeki İranlı Öğrencilerin } \\
\text { Yazma Problemleri } \\
\text { [The Writing Problems Of Iranian } \\
\text { Students in The Basic Level Who Learns } \\
\text { Turkish as a Foreign Language] }\end{array}$ & $\begin{array}{l}\text { Zeitschriftfürdie Welt } \\
\text { der Türken } \\
\text { [Journal of World of } \\
\text { Turks] }\end{array}$ & $6(2)$ & $335-349$ \\
\hline $\begin{array}{l}\text { Boylu, E. } \\
\text { Başar, U. }\end{array}$ & 2015 & $\begin{array}{l}\text { Yurt Dışında Türkiye Türkçesi Öğrenen } \\
\text { Türk Dillilerin Yazılı Anlatım Hataları: } \\
\text { İran Örneği } \\
\text { [Written Expression Mistakes Of Turkic } \\
\text { People Learning Turkish Outside Of }\end{array}$ & $\begin{array}{l}\text { Uluslararası Eğitim } \\
\text { Bilimleri Dergisi } \\
\text { [The Journal of } \\
\text { International } \\
\text { Educational Science] }\end{array}$ & 5 & $324-338$ \\
\hline
\end{tabular}


Boylu, E.

Güney, E. Z., Özyalçın, K. E
2017 Yanlış Çözümleme Yaklaşımına Göre Türkçeyi Yabancı Dil Olarak Öğrenen B1 Seviyesi Öğrencilerinin Yazılı Anlatımlarının Değerlendirilmesi [Evaluation of Written Expressions of B1 Students Learning Turkish as a Foreign Language According to Error Analysis Method]

\begin{tabular}{lll}
\hline Bölükbaş, F. $\quad 2011$ & Arap Öğrencilerin Türkçe Yazılı \\
& Anlatım Becerilerinin Değerlendirilmesi \\
& [An Evaluation of Arab Students’ \\
& Turkish Writing Skills]
\end{tabular}

\begin{tabular}{|c|c|c|c|c|c|}
\hline $\begin{array}{l}\text { Büyükikiz, K. } \\
\text { K. Hasırc1, S. }\end{array}$ & 2013 & $\begin{array}{l}\text { Yabancı Dil Olarak Türkçe Öğrenen } \\
\text { Öğrencilerin Yazılı Anlatımlarının } \\
\text { Yanlış Çözümleme Yaklaşımına Göre } \\
\text { Değerlendirilmesi } \\
\text { [Evaluation of Written Expressions of } \\
\text { Turkish Learners as a Foreign Language } \\
\text { According to Error Analysis Approach] }\end{array}$ & $\begin{array}{l}\text { Ana Dili Eğitimi } \\
\text { Dergisi-ADED } \\
\text { [Journal of Mother } \\
\text { Tongue Education- } \\
\text { JOMTE] }\end{array}$ & $1(4)$ & $51-62$ \\
\hline $\begin{array}{l}\text { Çerçi,A. } \\
\text { Derman, S. } \\
\text { Bardakçı, M }\end{array}$ & 2016 & $\begin{array}{l}\text { Yabancı Dil Olarak Türkçe Öğrenen } \\
\text { Öğrencilerin Yazılı Anlatımlarına } \\
\text { Yönelik Yanlış Çözümlemesi. } \\
\text { [An Error Analysis on TFL Learners' } \\
\text { Writings] }\end{array}$ & $\begin{array}{l}\text { Gaziantep University } \\
\text { Journal of Social } \\
\text { Sciences }\end{array}$ & $15(2)$ & $695-715$ \\
\hline Çetinkaya, G. & 2015 & $\begin{array}{l}\text { Yanlış Çözümlemesi: Yabancı Dil } \\
\text { Olarak Türkçe Öğrenen B2 Düzeyindeki } \\
\text { Öğrencilerin Yazılı Metinlerine İlişkin } \\
\text { Görünümler } \\
\text { [Error Analysis: The Views on Students' } \\
\text { Written Texts in Learning Turkish as a } \\
\text { Foreign Language at Level B2] }\end{array}$ & $\begin{array}{l}\text { International Journal } \\
\text { of Languages' } \\
\text { Education and } \\
\text { Teaching }\end{array}$ & $3(1)$ & $164-178$ \\
\hline $\begin{array}{l}\text { Gezer, H., } \\
\text { Kiymık, M. N }\end{array}$ & 2018 & $\begin{array}{l}\text { Türkçenin Yabancı Dil Olarak } \\
\text { Öğretiminde Arap Dilli Öğrencilerin } \\
\text { Yazılı Anlatım Becerilerinde } \\
\text { Karşılaştıkları Güçlüklere İlişkin Bir } \\
\text { Çözümleme } \\
\text { [An Analysis of Difficulties Confronted } \\
\text { in Written Expression Skills by Arabic } \\
\text { Language Students in Teaching Turkish } \\
\text { as Foreign Language] }\end{array}$ & $\begin{array}{l}\text { Uludağ Üniversitesi } \\
\text { Eğitim Fakültesi } \\
\text { Dergisi }\end{array}$ & 31 & $43-64$ \\
\hline İnan, $\mathrm{K}$. & 2014 & $\begin{array}{l}\text { Yabancı Dil Olarak Türkçe Öğrenen } \\
\text { İranlıların Yazılı Anlatımlarının Hata } \\
\text { Analizi Bağlamında Değerlendirilmesi } \\
\text { [An Evaluation of The Error Analysis in } \\
\text { The Written Expressions of Iranians } \\
\text { Who Learn Turkish as a Foreign } \\
\text { Language] }\end{array}$ & $\begin{array}{l}\text { Turkish Studies } \\
\text { International } \\
\text { Periodical For The } \\
\text { Languages, Literature } \\
\text { and History of Turkish } \\
\text { or Turkic }\end{array}$ & $9(9)$ & $619-649$ \\
\hline Kara, M. & 2010 & $\begin{array}{l}\text { Gazi Üniversitesi TÖMER } \\
\text { Öğrencilerinin Türkçe Öğrenirken }\end{array}$ & $\begin{array}{l}\text { Türk Eğitim Bilimleri } \\
\text { Dergisi }\end{array}$ & $8(3)$ & $661-696$ \\
\hline
\end{tabular}

\begin{tabular}{|c|c|c|c|c|c|}
\hline $\begin{array}{l}\text { Büyükikiz, K. } \\
\text { K. Hasırc1, S. }\end{array}$ & 2013 & $\begin{array}{l}\text { Yabancı Dil Olarak Türkçe Öğrenen } \\
\text { Öğrencilerin Yazılı Anlatımlarının } \\
\text { Yanlış Çözümleme Yaklaşımına Göre } \\
\text { Değerlendirilmesi } \\
\text { [Evaluation of Written Expressions of } \\
\text { Turkish Learners as a Foreign Language } \\
\text { According to Error Analysis Approach] }\end{array}$ & $\begin{array}{l}\text { Ana Dili Eğitimi } \\
\text { Dergisi-ADED } \\
\text { [Journal of Mother } \\
\text { Tongue Education- } \\
\text { JOMTE] }\end{array}$ & $1(4)$ & $51-62$ \\
\hline $\begin{array}{l}\text { Çerçi,A. } \\
\text { Derman, S. } \\
\text { Bardakçı, M }\end{array}$ & 2016 & $\begin{array}{l}\text { Yabancı Dil Olarak Türkçe Öğrenen } \\
\text { Öğrencilerin Yazılı Anlatımlarına } \\
\text { Yönelik Yanlış Çözümlemesi. } \\
\text { [An Error Analysis on TFL Learners' } \\
\text { Writings] }\end{array}$ & $\begin{array}{l}\text { Gaziantep University } \\
\text { Journal of Social } \\
\text { Sciences }\end{array}$ & $15(2)$ & $695-715$ \\
\hline Çetinkaya, G. & 2015 & $\begin{array}{l}\text { Yanlış Çözümlemesi: Yabancı Dil } \\
\text { Olarak Türkçe Öğrenen B2 Düzeyindeki } \\
\text { Öğrencilerin Yazılı Metinlerine İlişkin } \\
\text { Görünümler } \\
\text { [Error Analysis: The Views on Students' } \\
\text { Written Texts in Learning Turkish as a } \\
\text { Foreign Language at Level B2] }\end{array}$ & $\begin{array}{l}\text { International Journal } \\
\text { of Languages' } \\
\text { Education and } \\
\text { Teaching }\end{array}$ & $3(1)$ & $164-178$ \\
\hline $\begin{array}{l}\text { Gezer, H., } \\
\text { Kiymık, M. N }\end{array}$ & 2018 & $\begin{array}{l}\text { Türkçenin Yabancı Dil Olarak } \\
\text { Öğretiminde Arap Dilli Öğrencilerin } \\
\text { Yazılı Anlatım Becerilerinde } \\
\text { Karşılaştıkları Güçlüklere İlişkin Bir } \\
\text { Çözümleme } \\
\text { [An Analysis of Difficulties Confronted } \\
\text { in Written Expression Skills by Arabic } \\
\text { Language Students in Teaching Turkish } \\
\text { as Foreign Language] }\end{array}$ & $\begin{array}{l}\text { Uludağ Üniversitesi } \\
\text { Eğitim Fakültesi } \\
\text { Dergisi }\end{array}$ & 31 & $43-64$ \\
\hline İnan, $\mathrm{K}$. & 2014 & $\begin{array}{l}\text { Yabancı Dil Olarak Türkçe Öğrenen } \\
\text { İranlıların Yazılı Anlatımlarının Hata } \\
\text { Analizi Bağlamında Değerlendirilmesi } \\
\text { [An Evaluation of The Error Analysis in } \\
\text { The Written Expressions of Iranians } \\
\text { Who Learn Turkish as a Foreign } \\
\text { Language] }\end{array}$ & $\begin{array}{l}\text { Turkish Studies } \\
\text { International } \\
\text { Periodical For The } \\
\text { Languages, Literature } \\
\text { and History of Turkish } \\
\text { or Turkic }\end{array}$ & $9(9)$ & $619-649$ \\
\hline Kara, M. & 2010 & $\begin{array}{l}\text { Gazi Üniversitesi TÖMER } \\
\text { Öğrencilerinin Türkçe Öğrenirken }\end{array}$ & $\begin{array}{l}\text { Türk Eğitim Bilimleri } \\
\text { Dergisi }\end{array}$ & $8(3)$ & $661-696$ \\
\hline
\end{tabular}

International Journal 5(3) 184-202 of Languages'

Education and

Teaching

$\begin{array}{lll}\text { Turkish Studies } & 6(3) & 1357- \\ \text { International } & & 1367 \\ \text { Periodical For The } & & \\ \text { Languages, Literature } \\ \text { and History of Turkish } \\ \text { or Turkic }\end{array}$

\begin{tabular}{|c|c|c|c|c|c|}
\hline $\begin{array}{l}\text { Büyükikiz, K. } \\
\text { K. Hasırc1, S. }\end{array}$ & 2013 & $\begin{array}{l}\text { Yabancı Dil Olarak Türkçe Öğrenen } \\
\text { Öğrencilerin Yazılı Anlatımlarının } \\
\text { Yanlış Çözümleme Yaklaşımına Göre } \\
\text { Değerlendirilmesi } \\
\text { [Evaluation of Written Expressions of } \\
\text { Turkish Learners as a Foreign Language } \\
\text { According to Error Analysis Approach] }\end{array}$ & $\begin{array}{l}\text { Ana Dili Eğitimi } \\
\text { Dergisi-ADED } \\
\text { [Journal of Mother } \\
\text { Tongue Education- } \\
\text { JOMTE] }\end{array}$ & $1(4)$ & $51-62$ \\
\hline $\begin{array}{l}\text { Çerçi,A. } \\
\text { Derman, S. } \\
\text { Bardakçı, M }\end{array}$ & 2016 & $\begin{array}{l}\text { Yabancı Dil Olarak Türkçe Öğrenen } \\
\text { Öğrencilerin Yazılı Anlatımlarına } \\
\text { Yönelik Yanlış Çözümlemesi. } \\
\text { [An Error Analysis on TFL Learners' } \\
\text { Writings] }\end{array}$ & $\begin{array}{l}\text { Gaziantep University } \\
\text { Journal of Social } \\
\text { Sciences }\end{array}$ & $15(2)$ & $695-715$ \\
\hline Çetinkaya, G. & 2015 & $\begin{array}{l}\text { Yanlış Çözümlemesi: Yabancı Dil } \\
\text { Olarak Türkçe Öğrenen B2 Düzeyindeki } \\
\text { Öğrencilerin Yazılı Metinlerine İlişkin } \\
\text { Görünümler } \\
\text { [Error Analysis: The Views on Students' } \\
\text { Written Texts in Learning Turkish as a } \\
\text { Foreign Language at Level B2] }\end{array}$ & $\begin{array}{l}\text { International Journal } \\
\text { of Languages' } \\
\text { Education and } \\
\text { Teaching }\end{array}$ & $3(1)$ & $164-178$ \\
\hline $\begin{array}{l}\text { Gezer, H., } \\
\text { Kiymık, M. N }\end{array}$ & 2018 & $\begin{array}{l}\text { Türkçenin Yabancı Dil Olarak } \\
\text { Öğretiminde Arap Dilli Öğrencilerin } \\
\text { Yazılı Anlatım Becerilerinde } \\
\text { Karşılaştıkları Güçlüklere İlişkin Bir } \\
\text { Çözümleme } \\
\text { [An Analysis of Difficulties Confronted } \\
\text { in Written Expression Skills by Arabic } \\
\text { Language Students in Teaching Turkish } \\
\text { as Foreign Language] }\end{array}$ & $\begin{array}{l}\text { Uludağ Üniversitesi } \\
\text { Eğitim Fakültesi } \\
\text { Dergisi }\end{array}$ & 31 & $43-64$ \\
\hline İnan, $\mathrm{K}$. & 2014 & $\begin{array}{l}\text { Yabancı Dil Olarak Türkçe Öğrenen } \\
\text { İranlıların Yazılı Anlatımlarının Hata } \\
\text { Analizi Bağlamında Değerlendirilmesi } \\
\text { [An Evaluation of The Error Analysis in } \\
\text { The Written Expressions of Iranians } \\
\text { Who Learn Turkish as a Foreign } \\
\text { Language] }\end{array}$ & $\begin{array}{l}\text { Turkish Studies } \\
\text { International } \\
\text { Periodical For The } \\
\text { Languages, Literature } \\
\text { and History of Turkish } \\
\text { or Turkic }\end{array}$ & $9(9)$ & $619-649$ \\
\hline Kara, M. & 2010 & $\begin{array}{l}\text { Gazi Üniversitesi TÖMER } \\
\text { Öğrencilerinin Türkçe Öğrenirken }\end{array}$ & $\begin{array}{l}\text { Türk Eğitim Bilimleri } \\
\text { Dergisi }\end{array}$ & $8(3)$ & $661-696$ \\
\hline
\end{tabular}

Dergisi-ADED

[Journal of Mother

Tongue Education-

JOMTE]

[Evaluation of Written Expressions of

\section{(OMTE)}


Karşılaştıkları Sorunlar ve Bunların

Çözümüne Yönelik Öneriler

\begin{tabular}{|c|c|c|c|c|c|}
\hline $\begin{array}{l}\text { Nurlu, M. } \\
\text { Kutlu, A. }\end{array}$ & 2015 & $\begin{array}{l}\text { Türkçenin Yabancı Dil Olarak } \\
\text { Öğretiminde Temel Seviye A1 Yazma } \\
\text { Sorunları: Afganistan Örneği } \\
\text { [The Issue of A1 Level Spelling } \\
\text { Mistakes in Teaching Turkish as a } \\
\text { Foreign Language: The Case of } \\
\text { Afghanistan] }\end{array}$ & $\begin{array}{l}\text { Kara Harp Okulu } \\
\text { Bilim Dergisi } \\
\text { [Science Journal of } \\
\text { Turkish Military } \\
\text { Academy] }\end{array}$ & 2 & $67-87$ \\
\hline $\begin{array}{l}\text { Önder, A., } \\
\text { Uzdu-Yıldız, } \\
\text { F. }\end{array}$ & 2017 & $\begin{array}{l}\text { Türkçe Öğrenen Yabancıların Yazılı } \\
\text { Anlatım Yanlışlarının Çözümlenmesi } \\
\text { [The Analysis of the Writing Mistakes of } \\
\text { Foreigners Learning Turkish] }\end{array}$ & $\begin{array}{l}\text { International. Journal } \\
\text { of Languages' } \\
\text { Education and } \\
\text { Teaching }\end{array}$ & $5(4)$ & $100-114$ \\
\hline Subaşı, D. A. & 2010 & $\begin{array}{l}\text { TÖMER'de Yabancı Dil Olarak Türkçe } \\
\text { Öğrenen Arap Öğrencilerin } \\
\text { Kompozisyonlarında Hata Analizi } \\
\text { [Error Analysis on Composition of } \\
\text { Arabian Learners of Turkish at TÖMER] }\end{array}$ & Dil Dergisi & 148 & $7-16$ \\
\hline $\begin{array}{l}\text { Temizyürek, } \\
\text { F., Ünlü, H. }\end{array}$ & 2018 & $\begin{array}{l}\text { Türkiye Türkçesini Yabancı Dil Olarak } \\
\text { Öğrenen Gürcü Öğrencilerin Yazma } \\
\text { Becerisinde Karşılaştıkları Sorunlar ve } \\
\text { Çözüm Önerileri } \\
\text { [Problems in Writing Skills Faced by } \\
\text { Georgian Learners Of Turkish as a } \\
\text { Foreign Language and Suggested } \\
\text { Solutions] }\end{array}$ & $\begin{array}{l}\text { International Journal } \\
\text { of Languages' } \\
\text { Education and } \\
\text { Teaching }\end{array}$ & $6(1)$ & $316-327$ \\
\hline $\begin{array}{l}\text { Y1lmaz, F. } \\
\text { Bircan, D. }\end{array}$ & 2015 & $\begin{array}{l}\text { Türkçe Öğretim Merkezi'nde Okuyan } \\
\text { Yabancı Öğrencilerin Yazılı } \\
\text { Kompozisyonlarının "Yanlış } \\
\text { Çözümleme Yöntemi”ne Göre } \\
\text { Değerlendirilmesi } \\
\text { [Evaluation of the Essays Written by } \\
\text { Foreign Students Studying at Turkish } \\
\text { Language Center in Accordance with the } \\
\text { Error Analysis Method] }\end{array}$ & $\begin{array}{l}\text { International Journal } \\
\text { of Language Academy }\end{array}$ & $3(1)$ & $113-126$ \\
\hline
\end{tabular}

\subsection{Data analysis}

The articles that were included in the review were numbered to facilitate the process of analyzing the data and merging the findings. Each article was summarized under the following headings: the number of students, their native language, their country/nationality/continent, and their language level. A number of studies provided information on the native language of the sample while others presented information on the country or region. The information on participants of the studies is included in Table 4 as provided in the respective articles. 
Table 4. Information on Samples of Articles

\begin{tabular}{|c|c|c|c|c|}
\hline No & Sample Size & $\begin{array}{l}\text { Native } \\
\text { Language }\end{array}$ & Country/Nationality/Continent & Level \\
\hline 1 & 20 & Arabic & & $\begin{array}{l}\text { beginner, intermediate, } \\
\text { advanced }\end{array}$ \\
\hline 2 & 1324 & & $\begin{array}{l}\text { Africa, Central Asia, The Balkans and Middle } \\
\text { Eastern }\end{array}$ & \\
\hline 3 & 20 & Arabic & & \\
\hline$\overline{4}$ & 42 & & $\begin{array}{l}\text { Algeria, Comor Islands, Kongo, Lebanon, Iraq, } \\
\text { Egypt, Indonesia, Yemen, Nigeria, Senegal, } \\
\text { Somalia, Afghanistan, Syria }\end{array}$ & B2 \\
\hline 5 & 200 & & $\begin{array}{l}\text { Moldovya, Kosovo, Macedonia, Serbia, Bosnia- } \\
\text { herzegovina, Greece, Albania, Romania, } \\
\text { Montenegro, Crotia, Hungary }\end{array}$ & $\mathrm{A} 1, \mathrm{~A} 2, \mathrm{~B} 1, \mathrm{~B} 2, \mathrm{C} 1$ \\
\hline 6 & 71 & $\begin{array}{l}\text { Persian, } \\
\text { Azerbaijanese }\end{array}$ & Persian & B2 \\
\hline 7 & 120 & & Persian & \\
\hline 8 & 22 & & $\begin{array}{l}\text { Turkmenistan, Azerbaijan, Tajikistan, Kenya, } \\
\text { Afghanistan, Germany }\end{array}$ & A2 \\
\hline 9 & 50 & Arabic & & B2 \\
\hline 10 & 30 & & Persian & $\begin{array}{l}\text { Beginner and } \\
\text { intermediate }\end{array}$ \\
\hline 11 & 94 & & Afghan & A1 \\
\hline 12 & 14 & & $\begin{array}{l}\text { Afghanistan, Kenya, Pakistan, } \\
\text { Chad, Palestine, Gambia, Ghana, Rwanda, } \\
\text { Somali, Tunis ve Zambia, }\end{array}$ & $\mathrm{A} 1, \mathrm{~A} 2, \mathrm{~B} 1, \mathrm{~B} 2, \mathrm{C} 1$ \\
\hline$\overline{13}$ & 65 & & $\begin{array}{l}\text { Iraq, Ethiopia, the Democratic Republic of the } \\
\text { Congo, Sudan, Kazakstan, Uganda ve } \\
\text { Afghanistan Syria, Kenya, Eygpt, Persian, } \\
\text { Albania, Rusia, Cameroon, Morocco ve Eritrea, } \\
\text { Greece, Bangladesh, Vanuatu, Bosnia- } \\
\text { herzegovina, Mongolia, Georgia, Colombia, } \\
\text { Senegal, Sri Lanka, Montenegro, Serbia, } \\
\text { Tajikistan, Vietnam, Ukraine, Pakistan, Nigeria, } \\
\text { Niger, Nepal, Madagascar, Togo, Burkina Faso, } \\
\text { Zimbabwe }\end{array}$ & $\mathrm{A} 2, \mathrm{~B} 1, \mathrm{~B} 2$ \\
\hline$\overline{14}$ & 50 & & $\begin{array}{l}\text { Afghanistan, Syria, Palestine, Persian, Yemen, } \\
\text { Iraq, Kazakstan, China, Eygpt Tunis, Jordan }\end{array}$ & B1 \\
\hline 15 & & & Georgian & A1 \\
\hline 16 & 11 & Arabic & Iraq, Syria, Eygpt, Libya & B1 \\
\hline
\end{tabular}

It was observed that the researchers failed to use a common framework but opted for their own criteria to identify the steps of error analysis. Although this caused complications in converting the findings into numerical data, it did not pose an obstacle to the interpretation of tendencies in the errors made.

It was found that a number of titles for the same error type were named differently across articles; thus, the findings related to the same errors were merged. During this merging process, all publications were examined separately by two researchers and the results of these examinations were combined.

The articles examined differed in terms of the classification of the findings. Their classification of articles in terms of presenting their findings is included in Table 5. 
Table 5. Classification of Articles in Terms of Presentation of Findings

\begin{tabular}{lll}
\hline Classification & Article no & $\begin{array}{l}\text { Number } \\
\text { articles }\end{array}$ \\
\hline Providing numerical findings and examples based on classification & $3,4,5,8,9,12,13,14$ & 8 \\
\hline Providing only examples based on classification & $2,6,7,11,15,16$ & 6 \\
\hline Presenting findings with a comparative evaluation & 1,10, & 2 \\
\hline
\end{tabular}

As shown in Table 5, eight articles provided numerical findings with regard to the error rates and examples in their classification. Six articles included only examples, while two articles offered comments inquiring into the reasons of errors and their findings by making comparisons with the native language of the students.

The articles numbered $3,4,5,8,9,12,13$, and 14, which contained numerical findings in their classifications, were examined in terms of classification of their findings, contents, and examples, and then, the headings with the same content and similar examples were merged together. With regard to the type of classification, the articles numbered 3, 4, 5, 8, 9, 12, 13, and 14 showed similarities. In classifying the findings in the articles that showed similarities in terms of their contents and examples, four headings were selected on the basis of the general classifications in studies by Bölükbaş (2011); Büyükikiz and Hasırcı (2013); and Çerçi, Derman, and Bardakçı (2016). These headings were grammatical errors, syntactic errors, lexical errors, and spelling and punctuation errors. Grammatical errors include morphological errors as in "aileme çok özlüyordum" (Bölükbaş, 2011, p. 1362); syntactic errors contain word order errors as in "Türkiye çok bir güzel ülke" (Çerçi, Derman, \& Bardakçı, 2016, p. 707); lexical errors include errors related to the use of vocabulary elements as in "...çok hoşuma geldi"; and spelling and punctuation errors contain the errors about how words are spelled or how punctuation marks are used as in "bizim memlektta kızlar..." (Y1lmaz \& Bircan, 2015, p. 123).

Article numbers 1 and 10, which were not included in the common classification, contained examples of the errors that were believed to have been made by the students under the influence of their native language. Article number 1 provided examples of errors that were made under the influence of Arabic and Persian, while Article number 10 provided examples of errors made under the influence of Persian and Azerbaijani. Article 10 did not contain information on the rates of errors. As the headings included in the comparison did not overlap with the headings in other articles, it was not possible to include those headings in the general evaluation.

In the articles numbered $2,6,7,11,15$, and 16 , errors were listed depending on the examples encountered, and the rates for the classification of the errors were not included.

\section{Results}

The findings of the eight articles, the classification headings of which were found to overlap as a result of the examination, were evaluated in tandem. Classification headings were matched in the studies examined with content analysis. For instance, the heading "morphological" in article number 13 overlapped with the heading "grammar" in the articles numbered 3, 4, 5, 8 and 12, whereas the heading "phonological" matched with the content and examples under the heading "spelling and punctuation" in other articles in question. Thereafter, the findings containing numerical data on errors from the articles numbered $3,4,5,8,9,12,13$, and 14 were reviewed.

The level was not mentioned in article number 3. It was stated that the highest number of errors were made in spelling and punctuation, followed by grammar, lexical, and syntax. 
Article number 4 contained the findings of a study conducted with students at the B2 level. The frequency of errors was listed from the highest to the lowest as spelling and punctuation, grammar, lexical, and syntax.

Article number 5 analyzed the errors made at all levels in more detail when compared with other articles. Following the match of headings in this article with other articles, it was found that spelling and punctuation errors were the most frequent type of error, followed by grammar-, syntax-, and lexicalrelated errors.

Article number 8 covered the errors made by the students at the A2 level. This article reported the error frequency from the highest to the lowest as spelling and punctuation, grammar, lexical, and syntax.

Article number 9 offered findings related to the B2 level. It was stated that most of the errors were made in grammar, followed by syntax, spelling and punctuation, and lexical. In article number 9, the findings were also described in terms of linguistic, cognitive processing, and communicative characteristics.

Article number 12 contained findings related to all levels. Thus, this article listed the frequency of errors from the highest to the lowest as spelling and punctuation, grammar, syntax, and lexical at the A1 level; grammar, spelling and punctuation, lexical, and syntax at the A2 level; grammar, spelling and punctuation, syntax, and lexical at the B1 level; grammar, spelling and punctuation, lexical, and syntax at the B2 level; and grammar, spelling and punctuation, lexical, and syntax at the $\mathrm{C} 1$ level.

Article number 13 studied three levels: A2, B1, and B2. The frequency of errors from the highest to the lowest was listed in this article as spelling and punctuation, grammar, syntax and lexical at the A2 level; spelling and punctuation, grammar, syntax, and lexical at the B1 level; and spelling and punctuation, syntax, grammar, and lexical at the B2 level.

Article number 14 concentrated on the errors made at the A1, A2, and B1 levels. It was noted that most of the errors were made in lexical at the three levels, followed by grammar, spelling and punctuation, and syntax.

The findings obtained from the articles numbered 3, 4, 5, 8, 9, 12, 13 and 14 are summarized in Table 6 on the basis of language levels.

Table 6. Summary of Findings from the Articles Numbered 3, 4, 5, 8, 9, 12, 13, and 14

\begin{tabular}{lllll}
\hline Level & Grammar & Syntax & Lexical & $\begin{array}{l}\text { Spelling and } \\
\text { punctuation }\end{array}$ \\
\hline A1 & 20.67 & 13.69 & 9.89 & 56.27 \\
\hline A2 & 22.09 & 7.10 & 5.70 & 64.90 \\
\hline A2 & 45.84 & 10.23 & 10.95 & 33.35 \\
\hline A2 & 25.95 & 14.72 & 6.30 & 53.04 \\
\hline B1 & 50.96 & 6.45 & 14.90 & 38.70 \\
\hline B1 & 23.67 & 21.57 & 9.80 & 44.96 \\
\hline B2 & 31.00 & 9.90 & 14.40 & 44.40 \\
\hline B2 & 48.30 & 3.85 & 20.90 & 26.94 \\
\hline B2 & 20.85 & 33.11 & 6.61 & 39.42 \\
\hline B2 & 51.93 & 16.91 & 15.43 & 15.73 \\
\hline C1 & 45.43 & 9.09 & 18.45 & 27.03
\end{tabular}




\begin{tabular}{lllll} 
Not specified & 16.39 & 13.17 & 15.59 & 54.58 \\
\hline All levels & 44.35 & 6.13 & 2.48 & 47.03 \\
\hline A1, A2, B1 & 28.34 & 1.60 & 43.66 & 26.39 \\
\hline Average & 33.98 & 11.97 & 13.93 & 40.91 \\
\hline
\end{tabular}

\section{Language level-based evaluation}

The analysis of errors in relation to language levels revealed that the rate of the spelling and punctuation errors made by the students at the A1 level in article number 1 was $56.27 \%$ as shown in Table 6.

There were three articles that dealt with the written expression errors made by the students at the A2 level. The findings from these studies are presented in Table 7.

Table 7. Findings from Articles related to the A2 Level

\begin{tabular}{lllll}
\hline Level & Grammar & Syntax & Lexical & Spelling and punctuation \\
\hline A2 & 22.09 & 7.10 & 5.70 & 64.90 \\
\hline A2 & 45.84 & 10.23 & 10.95 & 33.35 \\
\hline A2 & 25.95 & 14.72 & 6.30 & 53.04 \\
\hline Average & 31.29 & 10.68 & 7.65 & 50.43 \\
\hline
\end{tabular}

An examination of Table 7 indicates that the highest rate of errors $(50.43 \%)$ made by the students at the A2 level was in spelling and punctuation. Similar to other levels, the lowest rate of errors $(7.65 \%)$ was in lexical.

At the B1 level, the highest rate of errors occurred (41.83\%) in spelling and punctuation, followed by grammatical errors $(37.32 \%)$ as provided in Table 8 .

Table 8. Findings from Articles Related to the B1 Level

\begin{tabular}{lcccc}
\hline Level & Grammar & Syntax & Lexical & $\begin{array}{l}\text { Spelling and } \\
\text { punctuation }\end{array}$ \\
\hline B1 & 50.96 & 6.45 & 14.90 & 38.70 \\
\hline B1 & 23.67 & 21.57 & 9.80 & 44.96 \\
\hline Average & 37.32 & 14.01 & 12.35 & 41.83 \\
\hline
\end{tabular}

Table 9. Findings from Articles related to the B2 Level

\begin{tabular}{lcccc}
\hline Level & Grammar & Syntax & Lexical & $\begin{array}{l}\text { Spelling and } \\
\text { punctuation }\end{array}$ \\
\hline B2 & 31.00 & 9.90 & 14.40 & 44.40 \\
\hline B2 & 48.30 & 3.85 & 20.90 & 26.94 \\
\hline B2 & 20.85 & 33.11 & 6.61 & 39.42 \\
\hline B2 & 51.93 & 16.91 & 15.43 & 15.73 \\
\hline Average & 38.02 & 15.94 & 14.34 & 31.62 \\
\hline
\end{tabular}


As presented in Table 9, errors were made most frequently in grammar $(38.02 \%)$ and not in spelling or punctuation at the $\mathrm{B} 2$ level.

One of the articles under the scope of the present study examined the errors made by students at the $\mathrm{C} 1$ level. According to the figures in Table 6, grammatical errors were the most frequently made errors by the students at the $\mathrm{C} 1$ level with a rate of $45.43 \%$.

One article did not specify the language level of the sample, while another article presented findings related to all levels. The articles in question reported spelling and punctuation errors as the most frequent errors with a rate of $54.58 \%$ and $47.03 \%$, respectively.

The article that contained findings related to the A1, A2, and B1 levels suggested that lexical errors were the most frequently made errors, with a rate of $43.66 \%$. The findings in that article differed from those in other articles.

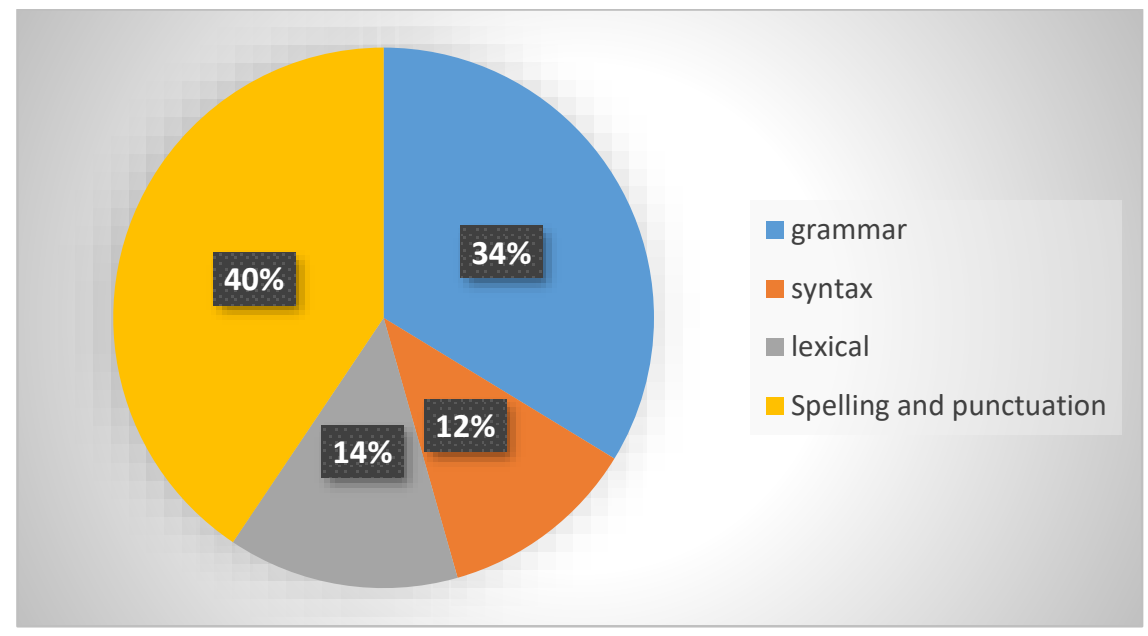

Figure 1. Average Error Rates at All Levels

Figure 1 includes the error rates for all levels based on the articles reviewed. As observable in Figure 1 , the most frequent error type was spelling and punctuation (40\%). Those errors were followed by grammatical errors (34\%). The errors related to lexical were found to have a rate of $14 \%$, followed by syntactic errors with $12 \%$. At all levels, lexical and syntax errors were less frequent when compared with other error types.

\section{Conclusions}

The present study, designed as a systematic review, produced findings that may be useful for researchers who plan to study the errors in written expressions made by learners of Turkish as a foreign language. The following conclusions were drawn on the basis of the findings:

- In terms of language levels, spelling and punctuation errors were higher than other types of errors at all levels.

- In terms of averages, the most frequent errors were the spelling and punctuation errors. However, when assessed on the basis of language level, the most frequent errors at the B2 and C1 levels were not about spelling and punctuation but about grammar. This implies that the spelling and punctuation errors decreased as the levels increased.

- The native languages of the samples were different; however, similar results were obtained across all samples. Even if the native language differed, the most frequently made errors were about spelling and punctuation. 
- In terms of sample size, the lowest number of participants was 11 and the highest number was 1,324. It was concluded that the error rates did not differ on the basis of sample size.

In this context, the error analysis studies conducted with learners of Turkish as a foreign language found that the students tended to make spelling and punctuation errors most frequently and that the rate of spelling and punctuation errors declined as the level of understanding of the target language increased.

To be able to make a more accurate interpretation with regard to the same results that were obtained with the students who had different native languages, further studies should be conducted with different participant groups, the members of which have the same or different native languages, and by using more specific error analysis classifications. This way, better evaluations of the effect of the native language can be made. Both the quality and quantity of studies in this field should be increased to obtain more generalizable results.

As in the work conducted by Tüm (2014) and Aydın and Gün (2018), determining the errors made by learners of Turkish as a foreign language in their verbal expressions in different samples and the systematic review of the findings of such studies may help to identify the problems encountered in teaching Turkish as a foreign language. In addition, studies that support the development of writing and speaking skills can be conducted by comparatively analyzing the errors made in written and verbal expressions.

When the error classification headings of the error analysis studies were considered, it was obvious that the headings, "grammatical errors" and "syntactic errors" did not provide thoroughly clear distinctions in terms of the Turkish language. The findings related to the spelling and punctuation errors contained examples regarding the wrong use of the case suffixes, and this made it harder to make a distinction between the headings "spelling and punctuation errors" and "grammatical errors." With regard to this, the study of new classification proposals for the assessment of the errors made by learners of Turkish as a foreign language will fill a significant niche in this field.

\section{Ethics Committee Approval}

The author(s) confirm(s) that the study does not need ethics committee approval according to the research integrity rules in their country (Date of Confirmation: December 11, 2019).

\section{References}

Ak Başoğul, D. \& Can, F. S. (2014). Yabancı Dil Olarak Türkçe Öğrenen Balkanlı Öğrencilerin Yazılı Anlatımda Yaptıkları Hatalar Üzerine Tespitler. Dil ve Edebiyat Eğitimi Dergisi, 10, 100-119.

Aydın, G. Gün, M. (2018). Çok Uluslu Aileye Sahip İki Dilli Çocukların Türkçe Sözlü Dil Becerilerinin Yanlış Çözümleme Yöntemine Göre İncelenmesi. Ana Dili Eğitimi Dergisi, 6(2), 325-342.

Boylu, E. (2014). Yabancı Dil Olarak Türkçe Öğrenen Temel Seviyedeki İranlı Öğrencilerin Yazma Problemleri. Zeitschriftfürdie Welt der Türken Journal of World of Turks, 6(2), 335-349

Boylu, E. Başar, U. (2015). Yurt Dışında Türkiye Türkçesi Öğrenen Türk Dillilerin Yazılı Anlatım Hataları: İran Örneği. Uluslararası Eğitim Bilimleri Dergisi, 5, 324-338

Boylu, E. Güney, E. Z., Özyalçın, K. E. (2017). Yanlış Çözümleme Yaklaşımına Göre Türkçeyi Yabanc1 Dil Olarak Öğrenen B1 Seviyesi Öğrencilerinin Yazılı Anlatımlarının Değerlendirilmesi. International Journal of Languages' Education and Teaching, 5(3), 184-202 
Bölükbaş, F. (2011). Arap Öğrencilerin Türkçe Yazılı Anlatım Becerilerinin Değerlendirilmesi. Turkish Studies - International Periodical For The Languages, Literature and History of Turkish or Turkic, 6(3), 1357-1367

Büyükikiz, K. K. Hasırc1, S. (2013). Yabancı Dil Olarak Türkçe Öğrenen Öğrencilerin Yazılı Anlatımlarının Yanlış Çözümleme Yaklaşımına Göre Değerlendirilmesi. Ana Dili Eğitimi Dergisi, $1(4), 51-62$

Büyüköztürk, Ş., Kılıç-Çakmak, E., Akgün, Ö. E., Karadeniz, Ş., Demirel F. (2015). Bilimsel Araştırma Yöntemleri. Pegem Akademi Yayınları: Ankara.

Corder, S. P. (1981). Error Analysis and Interlanguage. Oxford University Press.

Çalık, M., \& Sözbilir, M. (2014). Içerik Analizinin Parametreleri. Eğitim ve Bilim, 39, 33-38.

Çerçi,A. Derman, S. Bardakçı, M. (2016). Yabancı Dil Olarak Türkçe Öğrenen Öğrencilerin Yazılı Anlatımlarına Yönelik Yanlış Çözümlemesi. An Error Analysis on TFL Learners' Writings. Gaziantep University Journal of Social Sciences, 15(2), 695-715.

Çetinkaya, G. (2015). Yanlış Çözümlemesi: Yabanc1 Dil Olarak Türkçe Öğrenen B2 Düzeyindeki Öğrencilerin Yazılı Metinlerine İlişkin Görünümler. International Journal of Languages' Education and Teaching, 3(1), 164-178

Gass, S. M., Selinker, L. (2008). Second Language Acquisition: An Introductory Course. New York. Routledge.

Gezer, H., Kıymık, M. N. (2018). Türkçenin Yabancı Dil Olarak Öğretiminde Arap Dilli Öğrencilerin Yazılı Anlatım Becerilerinde Karşılaştıkları Güçlüklere İlişkin Bir Çözümleme. Uludağ Üniversitesi Eğitim Fakültesi Dergisi, 31, 43-64

Glass, G. V. (1976). Primary, Secondary and Meta-Analysis Of Research. The Educational Researcher, 10(5), 3-8.

İmer, K., Kocaman, A., Özsoy, A. S. (2011) Dilbilim Sözlüğ̈̈. İstanbul: Boğaziçi Üniversitesi Yayınları.

İnan, K. (2014). Yabancı Dil Olarak Türkçe Öğrenen İranlıların Yazılı Anlatımlarının Hata Analizi Bağlamında Değerlendirilmesi. International Periodical For The Languages, Literature and History of Turkish or Turki, 9(9), 619-649.

Kara, M. (2010). Gazi Üniversitesi TÖMER Öğrencilerinin Türkçe Öğrenirken Karşılaştıkları Sorunlar Ve Bunların Çözümüne Yönelik Öneriler. Türk Eğitim Bilimleri Dergisi, 8(3), 661-696.

Karaçam, Z. (2013). Sistematik Derleme Metodolojisi: Sistematik Derleme Hazırlamak için Bir Rehber. Dokuz Eylül Üniversitesi Hemşirelik Yüksekokulu Elektronik Dergisi, 6(1), 26-33.

Khansir, A. A., Ahrami, M. (2014). Error Analysis and Paragraph Writing. Language in India, 14(9), 74-162.

Nurlu, M. \&Kutlu, A. (2015). Türkçenin Yabancı Dil Olarak Öğretiminde Temel Seviye A1 Yazma Sorunları: Afganistan Örneği. Kara Harp Okulu Bilim Dergisi, 25(2), 67-87

Önder, A., Uzdu-Yıldız, F. (2017). Türkçe Öğrenen Yabancıların Yazılı Anlatım Yanlışlarının Çözümlenmesi International. Journal of Languages' Education and Teaching, 5(4), 100-114

Sridhar, S. N. (1981). Contrastive Analysis, Error Analysis, and Interlanguage: Three phases of one goal. In J. Fisiak (Ed.). Contrastive Linguistics and the Language Teacher. (pp. 207-241). Oxford: Pergamon Press. 
Subaşı, D. A. (2010). TÖMER'de Yabancı Dil Olarak Türkçe Öğrenen Arap Öğrencilerin Kompozisyonlarında Hata Analizi. Dil Dergisi, 148, 7-16

Temizyürek, F., Ünlü, H. (2018). Türkiye Türkçesini Yabancı Dil Olarak Öğrenen Gürcü Öğrencilerin Yazma Becerisinde Karşılaştıkları Sorunlar ve Çözüm Önerileri. International Journal of Language Academy, 6(1), 316-327

Tüm, G. (2014) Çok Uluslu Sinıflarda Yabancı Dil Türkçe Öğretiminde Karşılaşılan Sesletim Sorunları. Hacettepe Üniversitesi Eğitim Fakültesi Dergisi, 29(2), 255-266.

Yıldırım, A., Şimşek, H. (2013). Sosyal Bilimlerde Nitel Araştırma Yöntemleri. Ankara: Seçkin Yayınlar1

Yılmaz, F. Bircan, D. (2015). Türkçe Öğretim Merkezi’nde Okuyan Yabancı Öğrencilerin Yazılı Kompozisyonlarının "Yanlış Çözümleme Yöntemi"ne Göre Değerlendirilmesi. International Journal of Language Academy, 3(1), 113-126.

\section{Sistematik derleme çalışması: Yabancı dil olarak Türkçe öğrenenlerin yazılı anlatımlarındaki yanlışlar}

\section{$\ddot{O} \mathbf{z}$}

Bu çalışmada, yabancı dil olarak Türkçe öğrenenlerin yazılı anlatımlarında yaptıkları yanlışların çözümlenmesine yönelik araştırmalar sistematik derleme yöntemiyle incelenmiştir. Araştırmanın örneklemi alanyazın taraması sonucu ulaşılan 16 makaleyi kapsamaktadır. Makalelerin bulguları içerik analizi ile değerlendirilerek sistematik derleme oluşturulmuştur. Yabancı dil olarak Türkçe öğrenenlerin yazılı anlatımlarında yaptıkları yanlışları içeren 2010-2019 yılları arasında yayınlanmış, yayın dili Türkçe olan, örneklem grubu yetişkin ve yabancı dil olarak Türkçe öğrenicisi olan makaleler çalışmaya dahil edilmiştir. Derleme sonuçlarına göre yazılı dilde yabancı ögrencilerin en sık yaptıkları yanlışın \%40 oranı ile yazım ve noktalama başlıklarında toplandığı görülmektedir. Dilbilgisi yanlışları ise \%34 oranda yer almaktadır. \%14 oranıyla sözcük seçimi yanlışları görülürken sözdizim alanında yapılan yanlışlar ise $\% 12$ oranındadır. Tüm düzeylerde sözcük seçimi ve sözdizim yanlışları diğer alanlara oranla daha az yer almaktadır.

Anahtar sözcükler: Yabancı dil olarak Türkçe; yanlış; yanlış çözümleme; sistematik derleme; yazılı anlatım

\section{AUTHOR BIODATA}

Assist. Prof. Dr. Funda Uzdu Yıldız got her Master's Degree from Dokuz Eylul University, Teaching Turkish as a Foreign Language Department in 2008. She completed her Ph.D. study in Ege University, Department of Turkish Language and Literature, in 2012. She has been working in Dokuz Eylul University, Faculty of Literature, Linguistics Department since 2014. She is the Deputy Director of the Language Research and Teaching Center. Her professional interest areas are; language teaching, vocabulary teaching, material design.

Dr. Betül Çetin got her Master's Degree from Dokuz Eylul University, Teaching Turkish as a Foreign Language Department in 2008. She completed her Ph.D. study in Ankara University, Department of Linguistic, in 2015. She has been working in Dokuz Eylul University, Language Research and Teaching Center since 2009. She is the Deputy Director of the Language Research and Teaching Center. Her professional interest areas are; teaching Turkish as a language, grammar teaching, material design. 